\title{
Thrombotic Microangiopathy Syndrome in the ICU
}

\author{
S. Samy Modeliar, M. Monge, and M. Slama
}

\section{Introduction}

Since the first descriptions of haemolytic-uremic syndrome (HUS) by Moschowitz and thrombotic thrombocytopenic purpura (TTP) by Gasser, our knowledge about thrombotic microangiopathy (TMA) has grown considerably [1]. TMA now refers to a group of diseases comprising mechanical hemolytic anemia, peripheral thrombocytopenia, and varying degrees of organ failure. The incidence of TMA is increasing in the USA. Considerable progress has recently been made in the understanding of the pathophysiological mechanisms of TMA. These rare diseases, characterized by platelet thrombi in the microcirculation, are responsible for often serious organ dysfunction leading to the admission of these patients to intensive care units (ICUs). The prognosis of TMA was extremely poor prior to plasma therapy and especially plasma exchange. TMA is a serious, life-threatening disease that requires early diagnosis and urgent specialized therapeutic management.

\section{Definition}

TMA syndrome comprises a group of diseases characterized by a combination of: I mechanical hemolytic anemia

1 peripheral (consumption) thrombocytopenia

- varying degrees of organ failure.

Histologically, TMA is defined by the presence of thrombi in terminal arterioles and capillaries, responsible for varying degrees of organ failure.

The two main types of TMA are:

- TTP, characterized by frequently multiple organ involvement,

I HUS, in which renal involvement is predominant.

HUS and TTP share similar clinical features. Other forms of TMA are associated with multiple factors: cancers, certain chemotherapeutic agents, stem cell transplantation, human immunodeficiency virus (HIV) infection, malignant hypertension, pregnancy, post-partum, disseminated intravascular coagulation (DIC), HELLP syndrome (hemolysis, elevated liver enzymes and low platelets), giant hemangiomas and hemangio-endotheliomas. 


\section{Pathophysiology}

\section{General Description}

The early phenomenon common to all forms of TMA is damage to, or activation of, the vascular endothelium, responsible for local platelet aggregation, promoting the formation of platelet thrombi in the microcirculation. Various factors are responsible for this endothelial cell activation: infections, drugs, cancers, stem cell transplantation, etc. [2].

\section{Thrombotic Thrombocytopenic Purpura}

Role of von Willebrand factor (vWF) and ADAMTS-13 protein (A Disintegrin And Metalloprotease with ThromboSpondin type 1 motif). Physiologically, vWF is a multimeric glycoprotein that triggers the formation of platelet clot, and transport of clotting factor VIII. It is synthesized by megakaryocytes and endothelial cells, and then stored in endothelial and platelet Weibel-Palade bodies. Ultra-large vWF multimers (ULvWF) are composed of several vWF monomers linked by disulfide bonds. vWF multimers have more intense hemostatic properties than monomers [2].

The ADAMTS-13 protein is a metalloprotease that cleaves vWF multimers into monomers $[3,4]$. TTP is associated with very low levels of ADAMTS-13 protein activity $^{1}$, resulting in elevated plasma levels of ULvWF [5].

In this context of metalloprotease deficiency, ULvWF are released in response to endothelial cell damage, and then accumulate and adopt an optimal procoagulant configuration, resistant to the shearing forces of the microcirculation. ULvWF bind to platelet GPIIb and GPIIb/IIIa receptors, inducing excessive platelet aggregation. This mechanism is responsible for consumption thrombocytopenia and the formation of microthrombi decreasing the caliber of capillaries of the microcirculation, leading to tissue ischemia and erythrocyte fragmentation on thrombi (schistocytes) [2].

Deficiency of the protease cleaving ULvWF in TTP is attributed either to acquisition of an IgG inhibitory auto-antibody or to a mutation of the gene coding for the protease (familial cases) [6-10].

Role of infections in the pathogenesis of TTP. TMA is frequently associated with infection, whether or not there is another underlying disease [11]. In a retrospective intensive care study, $53 \%$ of cases of TMA were associated with infection [1]. These infections have a major impact on the patient's outcome [11]. Various microorganisms have been isolated during the acute phase of TTP, or during the days preceding TTP (Table 1 [12-20]). An underlying infection must be systematically sought in all cases of TMA.

The infectious agent involved in the pathogenesis of TTP induces a direct or indirect endothelial lesion (mediated by sepsis), as various mediators (interleukin [IL]-1 and IL-6, interferon gamma [IFN- $\gamma$, tumor necrosis factor [TNF]- $\alpha$ ) are released during the inflammatory reaction of sepsis and generate endothelial lesions.

${ }^{1}$ NB: ADAMTS-13 protein activity can be decreased in other pathological situations: DIC, ITPidiopathic thrombocytopenic purpura, systemic lupus erythematosus, cirrhosis, pregnancy, postoperatively, but it remains detectable in these situations, while it is virtually undetectable in TTP (activity $<5 \%$ ). 
Table 1. Non-exhaustive list of the main microorganisms isolated during TTP

\begin{tabular}{|c|c|c|}
\hline Bacteria & Viruses & Yeasts \\
\hline Pneumococcus & HIV & Cryptococcus \\
\hline Tuberculosis & Herpes & \\
\hline Borrelia & CMV & \\
\hline \multicolumn{3}{|l|}{ Legionella } \\
\hline \multicolumn{3}{|l|}{ Bacteroides } \\
\hline Rickettsia & & \\
\hline
\end{tabular}

HIV: human immunodeficiency virus; CMV: cytomegalovirus

Damaged endothelial cells degranulate, releasing procoagulant substances (ULvWF, platelet-activating factor [PAF]) into the plasma, express adhesion molecules and produce chemokines such as IL-8. These phenomena promote adhesion and activation of neutrophils, which participate in endothelial lesions. Activated endothelial cells also show decreased synthesis of prostaglandin I2 (PGI2), the most potent platelet aggregation inhibitor in the body. These mechanisms lead to a hypercoagulability state, which persists in individuals presenting predisposing factors.

\section{Hemolytic-uremic Syndrome}

The pathophysiology of HUS is characterized by intravascular coagulation specifically involving the renal microcirculation. Two very different forms are distinguished:

post-diarrheal HUS, usually endemic and mainly affecting young children (1-5 years),

- HUS occurring in the absence of diarrhea or atypical HUS, observed in older children and adults.

Post-diarrheal HUS [21]. This is the most frequent form of HUS (90\% of cases). The majority of cases are due to toxin-producing Escherichia coli gastrointestinal infection. The serotype most frequently isolated is E. coli $0157: \mathrm{H7}$, but many other serotypes as well as many other bacteria have been incriminated (Table 2). The E. coli toxin is called Shiga-like-toxin (SLT) due to its structural analogy with Shigella dysenteriae toxin type 1 .

The pathophysiology of post-diarrheal HUS starts with ingestion of food usually contaminated by a strain of toxin-producing $E$. coli. Intestinal colonization by $E$. coli is responsible for liquid diarrhea. This colonization phase is accompanied by massive toxin production. This toxin is released into the intestine and is responsi-

Table 2. Main bacteria involved in the pathogenesis of post-diarrheal HUS

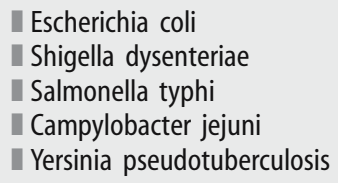


ble for direct microvascular and mucosal lesions, causing another episode of potentially hemorrhagic diarrhea. The toxin binds to specific receptors, is internalized and then enters the systemic circulation. E. coli bacteremia is not usually observed in post-diarrheal HUS. The toxin is transported by neutrophils to target tissues, i.e., the kidney, where it binds to specific receptors situated on endothelial cells of the renal cortex and medulla and tubular epithelial cells. It is internalized in renal cells and induces inhibition of protein synthesis via ribosomal inactivation (depurinization), leading to apoptotic cell death. In addition to these lesions, the toxin also stimulates cytokine production.

Endothelial cell damage has several consequences: expression of tissue factor on the cell surface, activation of coagulation, release of PAF into the circulation, and induction of overexpression of plasminogen activator inhibitor (PAI)-1 on the endothelial cell surface. Damaged endothelial cells also show decreased PGI2 production, and activate platelets and neutrophils predisposing to platelet aggregation on the endothelial cell surface. These phenomena lead to the formation of fibrin-rich microthrombi in renal capillaries, resulting in renal failure, formation of schistocytes and consumption thrombocytopenia.

Atypical HUS [2, 22]. Atypical HUS is much rarer (7 to $10 \%$ of cases), occurring sporadically in adults and older children. It is characterized by the absence of gastrointestinal infection. Its pathophysiology is poorly elucidated. Various hypotheses have been proposed in adults (viral infections, bacterial infections, certain drugs, etc.) to be responsible for endothelial lesions and features of HUS. A circulating factor present in the kidney has also been proposed.

Atypical HUS in children has been reported to be associated with persistent consumption of the $\mathrm{C} 3$ fraction of complement via the alternative pathway [23]. Decreased complement levels can be associated with factor $\mathrm{H}$ deficiency (factor $\mathrm{H}$ inhibits the alternative complement pathway). Atypical HUS with factor $\mathrm{H}$ deficiency usually corresponds to sporadic cases rather than familial cases and is rare in adults $[24,25]$. There is no clearly established correlation between factor $\mathrm{H}$ deficiency and the development of atypical HUS (factor $\mathrm{H}$ deficiency induces excessive C3 consumption, causing increased activation of neutrophils and excessive platelet aggregation).

HUS and pneumococcal infection (in children). This is a particular pathophysiological entity related to expression of the Thomson Friedenrich antigen on the surface of erythrocytes, endothelial cells, and glomeruli. This antigen, normally masked by sialic acid, is revealed by neuraminidase secreted by Pneumococcus. This antigen is then recognized by circulating IgM, leading to platelet aggregation and endothelial and glomerular lesions.

\section{- Diagnosis}

TMA disorders are rare in intensive care, with a prevalence of $0.35 \%$ of admissions according to a retrospective study conducted in adult intensive care units between 1998 and 2001 (64 patients) [11]. They represent 1 in 300 admissions, including cases of pre-eclampsia, stem cell transplantation, HELLP syndrome and terminal cancer. 
Table 3. Complementary investigations in TMA (non-exhaustive list, to be completed as a function of clinical data)

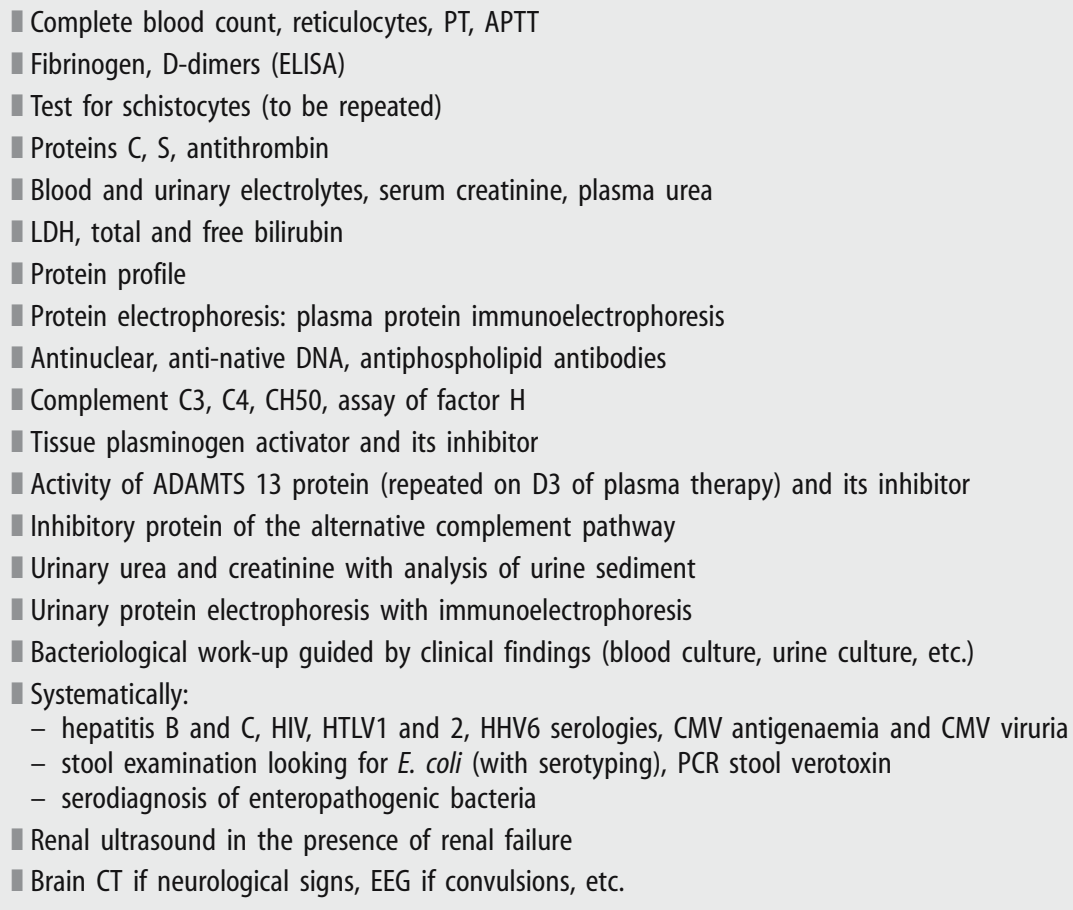

CT: computed tomography; EEG: electroencephalogram; HIV: human immunodeficiency virus; CMV: cytomegalovirus; PCR: polymerase chain reaction; HTLV: human T-cell leukemia virus; HHV: human herpesvirus

Early diagnosis is important (the prognosis is better when appropriate treatment is rapidly initiated). A diagnosis of TMA must be considered in any case of sudden onset of poorly defined neurological symptoms and hematological abnormalities (especially thrombocytopenia and anemia). TMA is observed in severely ill patients, often requiring admission to the ICU. In a retrospective study of TMA in 63 adult ICU patients conducted in 14 French teaching hospitals, the mean simplified acute physiology score (SAPS) II on admission was $45 \pm 26$ [11]. In another retrospective study, the mean SAPS II score on admission was $37 \pm 18$ (30 patients) [1].

The criteria for admission to the ICU are mainly life-threatening disease due to renal failure and/or neurological signs (convulsions, ischemia, coma). The other reasons for admission are hemorrhage, metabolic disorders (hepatocellular insufficiency), arrhythmias, myocardial infarction, etc [26].

\section{Clinical features of TTP $[2,27]$}

The clinical presentation of TTP is marked by a sudden onset in an adult in previously good health. It has a female predominance (M/F ratio: $3 / 2$ ) and generally occurs in the 4th decade. However, it can also occur in older or younger people. 
The estimated incidence is 4 per million inhabitants per year and has been constantly increasing over recent years. In $40 \%$ of cases, there is a prodromal phase resembling viral infection for several days before the acute episode (asthenia, arthralgia, myalgia, low back and abdominal pain). In $80 \%$ of cases, the triad suggestive of the diagnosis is observed immediately (mechanical hemolytic anemia, peripheral thrombocytopenia and neurological signs). In $40 \%$ of cases, two other cardinal signs are present (hyperthermia and renal failure). The diagnosis of TTP must always be considered in a patient with anemia and thrombocytopenia associated with organ failure.

Anemia is the most constant and earliest feature and consists of severe, regenerating (reticulocytes $>120,000 \mathrm{~mm}^{3}$ ), normochromic, normocytic, hemolytic (elevated lactate dehydrogenase $[\mathrm{LDH}]$ and bilirubin, and very low haptoglobin), microangiopathic anemia.

The blood smear reveals schistocytes, confirming the mechanical hemolysis (negative direct Coombs test). The presence of schistocytes may be delayed and should be looked for on subsequent smears [28]. Peripheral thrombocytopenia is marked (often less than $20,000 / \mathrm{mm}^{3}$ ) and constant and reflects disseminated intravascular platelet hyperaggregability. The hemorrhagic manifestations related to this thrombocytopenia are varied in terms of site and severity. Clotting tests are normal. Fibrin and fibrinogen degradation products (FDP) may be observed in rare cases.

Neurological signs are present in only $60 \%$ of cases at the initial phase of the disease (and globally in 84 to $92 \%$ of cases during the course of the disease). They are characterized by their sudden onset and transient nature, and intermittent involvement of various territories over an interval of several hours. Neurological signs comprise: obtundation, confusion, hemiparesis, dysarthria, aphasia, coma, disorders of consciousness, sensory deficit. Deep tendon reflexes are often brisk. Generalized convulsions are observed in $20 \%$ of cases and can evolve to status epilepticus.

Hyperthermia is present in $20 \%$ of cases at the initial phase, and then in 59 to $89 \%$ of cases during the course of the disease. It is usually only low-grade and reflects underlying hemolysis and cellular ischemia.

Renal failure occurs in one half of cases. It is usually moderate and, in the majority of cases, consists of macroscopic hematuria or slight proteinuria (less than $3 \mathrm{~g} / 24 \mathrm{~h}$ ). Oligo-anuric renal failure is rare.

Other organ lesions may be observed, particularly myocardial infarction or myocarditis that may lead to acute cardiogenic shock and chronic heart failure. Acute respiratory distress syndrome (ARDS) requiring mechanical ventilation, colonic ischemic, acute pancreatitis and sometimes ocular lesions may also be observed.

Sporadic TTP that resolves definitively is distinguished from recurrent forms with regular and frequent relapses, and intermittent TTP associated with irregular relapses [29].

\section{Clinical Features of HUS}

HUS comprises a combination of mechanical hemolytic anemia, peripheral thrombocytopenia and acute renal failure. The renal lesion is predominant in all forms and is classically associated with severe hypertension at the time of diagnosis. This hypertension is more severe and more frequent than during TTP, while neurological signs are less frequent than during TTP. 
Anemia and thrombocytopenia have similar characteristics to those of TTP (see above). Thrombocytopenia can be more severe than during TTP. Clotting abnormalities may be observed: slight reduction of fibrinogen and clotting factors $\mathrm{V}$ and VII. Elevation of fibrin degradation products, tissue plasminogen activator and PAI reflect activation of homeostasis by tissue factor in the kidney.

Kidney needle biopsy is only performed when there is a doubt about the diagnosis or in the case of persistent renal failure [30].

Epidemic (post-diarrheal) HUS. Post-diarrheal HUS has a sudden onset in $90 \%$ of cases, presenting with diarrhea (bloody in $70 \%$ of cases and febrile in $50 \%$ of cases). It is essentially observed in children, but also in adults. The renal lesion appears one week after onset of the diarrhea (which has resolved at the time of diagnosis in $50 \%$ of cases). Clinical manifestations are dominated by renal failure with severe proteinuria and hematuria (microscopic or macroscopic). Fifty percent of children are anuric at the time of diagnosis. One half of patients with renal failure will require dialysis. Severe hypertension is often associated with marked hyponatremia. Central nervous system signs are present in 10 to $30 \%$ of cases, often accentuated by hyponatremia, consisting of irritability, drowsiness, convulsions or even coma. The microorganism responsible is often no longer present in the stools at the time of the diagnosis of HUS, but the toxin can be isolated in two thirds of cases. The clinical and laboratory diagnosis of HUS is usually easy to establish and kidney needle biopsy is rarely necessary.

Atypical HUS. The clinical features are often less typical, and kidney needle biopsy is more often required to establish the diagnosis. Acute renal failure is often anuric with moderate proteinuria ( 1 to $2 \mathrm{~g} / 24 \mathrm{~h}$ ), but proteinuria may also exceed $3 \mathrm{~g} / 24 \mathrm{~h}$. Microscopic hematuria is frequent and hypertension is present in $50 \%$ of cases at diagnosis. Thrombocytopenia is present in only $50 \%$ of cases at diagnosis. There are few or no extra-renal signs (fever, neurological signs). A history of infection is frequently reported. Adults sometimes present a context of auto-immune disease, drugs or pregnancy.

The assessment of atypical HUS must include assays of complement fractions (C3, C4, CH50), and the activity of the ADAMTS-13 protein and the inhibitory protein of the alternative complement pathway (and a test for a mutation of the corresponding gene).

The clinical features of HUS and TTP tend to overlap and it can be difficult, on admission, to distinguish between these two diagnoses. The neurological signs are variable, ranging from simple confusion to coma with generalized convulsions and focal deficits, aphasia, diplopia, facial paralysis, etc. Renal involvement is also heterogeneous, ranging from normal renal function to anuric acute renal failure. Laboratory test results can confirm the diagnosis, but often only retrospectively.

\section{Prognosis}

The prognosis of TMA prior to plasma therapy in the ICU was extremely poor. Plasma therapy has considerably improved the prognosis of these usually fatal diseases (3-month mortality greater than $90 \%$ for acute forms of TTP without treatment) [27]. In a recent retrospective study of 64 patients admitted to the adult ICU for TMA, the mortality rate was 35\% [11] and Coppo et al. reported a mortality rate of $25 \%$ in intensive care patients with TMA [1]. 
Table 4. Main non-infectious circumstances (triggering factors or etiologies?) associated with TMA

I Pregnancy: antepartum and post-partum

- Cancers, hemangioma, hemangio-endothelioma

I Solid organ transplantation (kidneys, liver), hematopoietic stem cell transplantation

- Connective tissue diseases:

- polyarteritis nodosa

- systemic lupus erythematosus

- acute scleroderma

- ankylosing spondylitis

- Sjögren syndrome

Drugs:

- penicillin, mitomycin C, cyclosporine

- heparin, lidocaine, penicillamine D

- tacrolimus, oral contraceptives, quinine, ticlopidine (non-exhaustive list)

Ioxins:

- cocaine

The mortality related to TMA in intensive care is partly dependent on the treatment used. In recent studies [31-33], the survival rate of patients treated with plasma exchange is 78 to $90 \%$. In the study by Pene et al. [11], the mortality at 90 days was $22 \%$ when plasma exchange was performed and $59 \%$ in the absence of plasma exchange. The study by Rock et al. [33] confirmed the superiority of plasma exchange compared to fresh frozen plasma (FFP) infusion in terms of survival and response to treatment (78 versus $49 \%$ ). The use of plasma exchange is positively correlated with survival.

Overall, TMA syndromes with severe organ dysfunction requiring admission to the ICU are associated with a high mortality. The main cause of death in these patients in the ICU is multiple organ failure (MOF). An infection associated with TMA syndrome has a major impact on the outcome of these patients. The presence of a neurological deficit, possibly evaluated by the Glasgow Coma Scale, is the main negative prognostic factor correlated with mortality $[11,32,34,35]$. Other prognostic factors have also been demonstrated: the SAPS II score, need for vasoactive support, bilirubin level, LDH kinetics on the 3rd day of treatment [11]. Predictive scores for TTP have been established, but their value has not yet been validated [35].

Unlike other diseases usually observed in intensive care, the development of acute renal failure or the need for dialysis in a context of TMA are not correlated with mortality [11, 33, 36]. According to Lara et al. [32], renal failure is correlated with a risk of relapse of TMA, which has a poor prognosis, but the renal prognosis of TTP is generally good.

The survival rates of post-diarrheal HUS are excellent in response to symptomatic treatment alone, and admission to the ICU is only rarely necessary. The mortality of post-diarrheal HUS varies between 3 and $8 \%$ and is related to gastrointestinal and neurological lesions. The short-term renal prognosis is good (improvement of renal function over several days to several weeks). However, one quarter to one third of children still present renal sequelae 10 years later (proteinuria and/or renal failure) with end-stage chronic renal failure in 3 to $4 \%$ of cases. 
Atypical HUS in children has a poor prognosis, frequently resulting in end-stage chronic renal failure and a high post-transplantation recurrence rate. Atypical HUS in adults is a serious disease in which the prognosis depends on the etiology of HUS. The concomitant presence of advanced cancer, HIV infection, mitomycin $\mathrm{C}$ therapy and post-partum etiology carries a very poor prognosis. The intensive care mortality rate of atypical HUS is 10 to $20 \%$. Twenty-five to $50 \%$ of these patients will require chronic dialysis. Only $50 \%$ of survivors are completely cured without sequelae. In patients with underlying nephropathy, chronic renal failure persists after the acute episode in $70 \%$ of cases. Long-term recurrences are possible, especially when the cause of HUS persists, but are less frequent than for TTP.

\section{Management}

TMA always requires emergency treatment. Admission to intensive care, in addition to the usual criteria of organ failure, must be proposed in all patients with severe thrombocytopenia (less than $50,000 / \mathrm{mm}^{3}$ ), due to the high frequency of organ dysfunction at the acute phase of the disease.

\section{Symptomatic Treatment}

Symptomatic treatment is required in all forms of TMA. TMA disorders always have an unpredictable course. All available measures of 'aggressive intensive care' must, therefore, be proposed, even in the case of severe neurological signs.

Patients with respiratory failure require mechanical ventilation. This corresponds to $59 \%$ of all patients admitted to the ICU for TMA [11]. When possible, non-invasive ventilation should be preferred to invasive ventilation due to the hemorrhagic and infectious risks associated with invasive ventilation. Patients with severe renal failure require dialysis (47\% of TMA patients in intensive care) and $32 \%$ of patients require vasopressor support. Hypertension must also be treated, preferably by angiotensin converting enzyme (ACE) inhibitors. The target blood pressure is $120 / 80$ mmHg.

Folate supplementation is systematically prescribed (intense folate-consuming bone marrow regeneration). Anemia must be corrected by packed cell transfusion to achieve hemoglobin concentrations greater than $8 \mathrm{~g} / \mathrm{dl}$. Platelet transfusions should be avoided, except in the case of uncontrolled bleeding, as they may increase the thrombotic process in the microcirculation. Anticonvulsants should be prescribed to patients with a history of epilepsy. Any triggering or associated factors must be treated. In particular, antibiotics should be initiated whenever an infection is suspected (except for cases of post-diarrheal HUS, in which antibiotics can worsen the HUS [37]). Persistence of an occult bacterial infection can lead to persistence of TTP, making it refractory to conventional treatment.

\section{Specific Treatment: Plasma Therapy}

Indication. This treatment is not indicated in post-diarrheal HUS, as plasma therapy does not modify survival (which is excellent in response to symptomatic treatment alone). Plasma therapy must be urgently initiated in patients with TTP, as its efficacy in terms of survival has been demonstrated. Plasma therapy is used in adults 
with HUS by analogy with the management with TTP, although no studies are in favor of either plasma exchange or FFP transfusion. It is essential to control underlying predisposing factors.

Fresh frozen plasma transfusion or plasma exchange? Recent studies have demonstrated the superiority of plasma exchange over FFP transfusion in terms of survival and response to treatment of TTP [36]. The use of plasma exchange is independently correlated with survival. The efficacy of plasma exchange depends on the volume of plasma administered to the patient (plasma exchange is able to administer three times more plasma than FFP transfusion alone [11]). Two pathophysiological mechanisms can explain the efficacy of plasma exchange: plasma exchange removes a 'pathogenic' component from the patient's plasma (ULvWF or auto-antibodies) and compensates for the deficient protease (by providing large quantities of plasma). In cases of TTP not associated with an inhibitor, FFP transfusions may be sufficient. When plasma exchange cannot be started immediately (for technical reasons, or while waiting for transfer to a specialized center), large-volume FFP transfusion ( 20 to $30 \mathrm{ml} / \mathrm{kg} /$ day) can be started prior to plasma exchange, but it is associated with a risk of fluid overload, protein-overload proteinuria, hyperproteinemia, and hyperviscosity syndrome. The raised oncotic pressure can also accentuate renal insufficiency.

The plasma exchange technique is based on the daily exchange of one to two plasma masses fully compensated by FFP. A plasma exchange session lasts about 3 hours for 50 to $80 \mathrm{ml} / \mathrm{kg}$ of FFP. It is performed via a central venous catheter, rarely placed in a subclavian vein due to the risk of bleeding, and requires anticoagulation at effective doses during the session. A systematic calcium supplement is started during plasma exchange (risk of hypocalcemia due to the toxicity of citrate).

Plasma exchange is time-consuming, expensive and invasive and carries a risk of immunological pulmonary edema (almost eliminated by solvent/detergenttreated plasma). The duration of treatment is variable. Plasma exchange is continued daily until restoration of a normal platelet count $\left(>150,000 / \mathrm{mm}^{3}\right)$ for at least 48 hours with a reduction in LDH levels and reticulocyte counts. The frequency of plasma exchange must be progressively decreased. Daily plasma exchange must be reintroduced at the slightest sign of relapse. In the future, monitoring of ADAMTS13 protein inhibitor levels could guide the frequency of plasma exchange.

During relapses ( $30 \%$ of cases of TTP), the initial treatment in the acute phase is the same as for the first episode. Repeated relapses may represent an indication for splenectomy, during a period of remission. Refractory TTP, defined by no improvement of the platelet count on day 5 of treatment, requires twice-daily plasma exchange and adjuvant therapies (vincristine, polyvalent immunoglobulins, cyclophosphamide: see below) [38, 39].

\section{Second-line Adjuvant Therapy}

High-dose corticosteroid therapy is effective in $56 \%$ of purely hematological forms of TTP [31], although its efficacy has not been clearly demonstrated by randomized trials. In the absence of a contraindication (active infection), and despite a low level of evidence, methylprednisolone ( $1 \mathrm{mg} / \mathrm{kg} / \mathrm{day}$ for 3 weeks) can be administered immediately following plasma exchange.

The therapeutic value of platelet aggregation inhibitors, vincristine, high-dose immunoglobulins, splenectomy, immunosuppressives, unfractionated heparin, fibri- 
nolytics, prostacyclin, and vitamin $\mathrm{E}$ in the intensive care management of TMA has not been demonstrated.

Staphylococcal protein A columns could be effective in TTP, especially in a context of cancer, but they have not been evaluated in patients with an inhibitor [40]. A possible treatment for the future would be purified or recombinant protease infusion, which could replace plasma therapy.

\section{Other TMA syndromes}

\section{Pregnancy-associated TMA}

Various types of TMA can occur during pregnancy and the post-partum period: TTP, HUS, HELLP syndrome. Many complications have been described including acute necrotic pancreatitis, myocardial infarction, gastrointestinal ischemia, etc. The treatment of TTP during pregnancy is based on plasma exchange. Pregnancy does not modify the response to treatment, but the consequences of plasma exchange on the fetus have not been evaluated. There is a risk of relapse during subsequent pregnancies.

Ante-partum HUS requires termination of pregnancy, but has a fairly good prognosis. Post-partum HUS can occur up to 3 months after delivery and has a poorer prognosis. Death may be due to cerebral ischemic or hemorrhagic lesions or cardiac sudden death. Some cases of post-partum HUS are related to factor $\mathrm{H}$ deficiency (heterozygous mutation).

The HELLP syndrome (hepatic form of TMA) is a TMA disorder specific to pregnancy. It differs from HUS and TTP by the presence of DIC and liver impairment. Treatment consists of fetal extraction. Plasma therapy has been proposed ( 3 to 5 post-partum sessions appear to be effective). There is a risk of recurrence during subsequent pregnancies. These patients should preferably be treated in a specialized center.

\section{Hematopoietic Stem Cell Transplantation-associated TMA}

In this context, TMA is triggered by various predisposing factors including total body irradiation, infections (immunodepressed patients), drugs (tacrolimus, cyclosporine A), and acute graft versus host disease. These predisposing factors induce disseminated endothelial damage. The ADAMTS-13 protein level is normal and the response to treatment is disappointing. Plasma exchange improves the prognosis, which nevertheless remains poor. Management of predisposing factors is an essential aspect of treatment.

\section{Cancer-associated TMA}

TMA is essentially associated with secretory adenocarcinomas, such as breast or stomach cancers, or more rarely lung, colon or prostate cancers. Stomach cancers represent more than $50 \%$ of all reported cases of cancer-associated TMA. In most cases, the cancer has already been diagnosed and treated, but TTP can also be the first sign of cancer. The pathophysiology of cancer-associated TMA has been poorly elucidated and is associated with variable ADAMTS-13 protein levels. The prognosis is also variable and depends on the underlying cancer. 


\section{Drug- or Toxin-associated TMA}

Many drugs have been incriminated or suspected including ticlopidine, clopidogrel, cyclosporine A, tacrolimus, interferon alpha, oral contraceptives, quinine, cisplatin, mitomycin $\mathrm{C}$, bleomycin, arsenic, penicillamine $\mathrm{D}$.

Ticlopidine and clopidogrel are associated with severe ADAMTS-13 protein deficiency with the presence of plasma inhibitors. Plasma exchange therapy achieves complete remission in the majority of these patients [41, 42].

TMA associated with mitomycin $\mathrm{C}$ is dose-dependent and is observed after a total dose of more than $60 \mathrm{mg}$ ( 2 to $10 \%$ of treated patients). The TMA disorders often appear several months after stopping mitomycin $\mathrm{C}$ and are characterized by hypertension and pulmonary edema [43]. Neurological disorders and hyperthermia are rarely present. The prognosis is very serious despite discontinuation of mitomycin and treatment by plasma exchange (mortality of 60 to $70 \%$ ).

\section{HIV-associated TMA [12]}

These forms of TMA generally occur at an advanced stage of HIV infection and, therefore, have a very poor prognosis. Various mechanisms can be responsible:

- development of anti-ADAMTS-13 auto-antibodies responsible for TTP that responds favorably to treatment

- a multifactorial origin (opportunistic CMV infection [44]), drugs (valaciclovir, etc.) with a more variable response to treatment and a poorer prognosis.

Treatment of this type of TMA syndrome must be associated with antiretroviral therapy.

\section{Other TMA syndromes}

Other diseases have also been associated with TMA disorders, including catastrophic antiphospholipid syndrome, veno-occlusive disease, DIC, type 2 heparininduced thrombocytopenia, giant hemangioma, hemangio-endothelioma, and malignant hypertension. These various syndromes must be eliminated before initiating plasma therapy.

\section{Conclusion}

Major studies designed to improve our understanding of the pathophysiology of TMA have been conducted over recent years. This improved knowledge opens up new perspectives for more targeted treatment. However, until these innovative treatments become available, early diagnosis of these diseases is essential in order to rapidly initiate specific treatment, as the interval between diagnosis and initiation of plasma exchange is a decisive element in the prognosis of TTP. Treatment must not be stopped too early or too rapidly and must take into account the various associated factors, especially the presence of infection. 


\section{References}

1. Coppo P, Adrie C, Azoulay E, et al (2003) Infectious diseases as a trigger in thrombotic microangiopathies in intensive care unit (ICU) patients? Intensive Care Med 29:564-569

2. Coppo P, Vernant J, Veyradier A, et al (2005) Thrombotic thrombocytopenic purpura and other thrombotic microangiopathy syndromes. EMC-Hématologie 2:14-34

3. Fujikawa K, Suzuki H, McMullen B, Chung D (2001) Purification of human von Willebrand factor-cleaving protease and its identification as a new member of the metalloproteinase family. Blood 98:1662-1666

4. Gerritsen HE, Robles R, Lammle B, Furlan M (2001) Partial amino acid sequence of purified von Willebrand factor-cleaving protease. Blood 98:1654-1661

5. Moake JL (2002) Thrombotic microangiopathies. N Engl J Med 347:589-600

6. Furlan M, Robles R, Galbusera M, et al (1998) von Willebrand factor-cleaving protease in thrombotic thrombocytopenic purpura and the hemolytic-uremic syndrome. N Engl J Med 339:1578-1584

7. Levy GG, Nichols WC, Lian EC, et al (2001) Mutations in a member of the ADAMTS gene family cause thrombotic thrombocytopenic purpura. Nature 413:488-494

8. Remuzzi G, Galbusera M, Noris M, et al (2002) von Willebrand factor cleaving protease (ADAMTS13) is deficient in recurrent and familial thrombotic thrombocytopenic purpura and hemolytic uremic syndrome. Blood 100:778-785

9. Tsai HM, Lian EC (1998) Antibodies to von Willebrand factor-cleaving protease in acute thrombotic thrombocytopenic purpura. N Engl J Med 339:1585-1594

10. Veyradier A, Obert B, Houllier A, et al (2001) Specific von Willebrand factor-cleaving protease in thrombotic microangiopathies: a study of 111 cases. Blood 98:1765-1772

11. Pene F, Vigneau C, Auburtin M, et al (2005) Outcome of severe adult thrombotic microangiopathies in the intensive care unit. Intensive Care Med 31:71-78

12. Hymes KB, Karpatkin S (1997) Human immunodeficiency virus infection and thrombotic microangiopathy. Semin Hematol 34:117-125

13. Jeejeebhoy FM, Zaltzman JS (1998) Thrombotic microangiopathy in association with cytomegalovirus infection in a renal transplant patient: a new treatment strategy. Transplantation 65:1645-1648

14. Miniero R, Nesi F, Vai S, et al (1997) Cryptococcal meningitis following a thrombotic microangiopathy in an unrelated donor bone marrow transplant recipient. Pediatr Hematol Oncol 14:469-474

15. Moorthy B, Makker SP (1979) Hemolytic-uremic syndrome associated with pneumococcal sepsis. J Pediatr 95:558-559

16. Riggs SA, Wray NP, Waddell CC, Rossen RD, Gyorkey F (1982) Thrombotic thrombocytopenic purpura complicating Legionnaires' disease. Arch Intern Med 142:2275-2280

17. Satoh K, Takahashi H, Nagai K, Shibata A (1988) Thrombotic thrombocytopenic purpura and herpes zoster infection. Ann Intern Med 108:154-155

18. Schroder S, Spyridopoulos I, Konig J, Jaschonek KG, Luft D, Seif FJ (1995) Thrombotic thrombocytopenic purpura (TTP) associated with a Borrelia burgdorferi infection. Am J Hematol 50:72-73

19. Shalev O, Karni A, Kornberg A, Brezis M (1981) Thrombotic thrombocytopenic purpura associated with Bacteroides bacteremia. Arch Intern Med 141:692

20. Toscano V, Bontadini A, Falsone G, et al (1995) Thrombotic thrombocytopenic purpura associated with primary tuberculosis. Infection 23:58-59

21. Noris M, Remuzzi G (2005) Hemolytic uremic syndrome. J Am Soc Nephrol 16:1035-1050

22. Rossert J, Fischer E (2001) Insuffisances rénales aiguës d'origine glomérulaire et vasculaire. In: Offenstadt G, Vachon F, Cardinaud JP, et al (eds) Réanimation Médicale. Masson, Paris, pp 1239-1248

23. Roodhooft AM, McLean RH, Elst E, et al (1990) Recurrent haemolytic uraemic syndrome and acquired hypomorphic variant of the third component of complement. Pediatr Nephrol 4:597-599

24. Dragon-Durey MA, Fremeaux-Bacchi V, Loirat C, et al (2004) Heterozygous and homozygous factor $\mathrm{h}$ deficiencies associated with hemolytic uremic syndrome or membranoproliferative glomerulonephritis: report and genetic analysis of 16 cases. J Am Soc Nephrol 15:787-795 
25. Dragon-Durey MA, Loirat C, Cloarec S, et al (2005) Anti-Factor H autoantibodies associated with atypical hemolytic uremic syndrome. J Am Soc Nephrol 16:555-563

26. Knobl P, Rintelen C, Kornek G, et al (1997) Plasma exchange for treatment of thrombotic thrombocytopenic purpura in critically ill patients. Intensive Care Med 23:44-50

27. Tardy B (2001) Purpura thrombotique thrombocytopénique. In: Offenstadt G, Vachon F, Cardinaud JP, et al (eds) Réanimation Médicale. Masson, Paris, pp 1316-1319

28. Remuzzi G, Ruggenenti P (1995) The hemolytic uremic syndrome. Kidney Int 48:2-19

29. Sadler JE, Moake JL, Miyata T, George JN (2004) Recent advances in thrombotic thrombocytopenic purpura. Hematology (Am Soc Hematol Educ Program):407-423

30. Chandler WL, Jelacic S, Boster DR, et al (2002) Prothrombotic coagulation abnormalities preceding the hemolytic-uremic syndrome. N Engl J Med 346:23-32

31. Bell WR, Braine HG, Ness PM, et al (1991) Improved survival in thrombotic thrombocytopenic purpura-hemolytic uremic syndrome. Clinical experience in 108 patients. N Engl J Med 325:398-403

32. Lara PN, Jr, Coe TL, Zhou H, et al (1999) Improved survival with plasma exchange in patients with thrombotic thrombocytopenic purpura-hemolytic uremic syndrome. Am J Med 107:573-579

33. Rock G, Shumak K, Kelton J, et al (1992) Thrombotic thrombocytopenic purpura: outcome in 24 patients with renal impairment treated with plasma exchange. Canadian Apheresis Study Group. Transfusion 32:710-714

34. Pereira A, Mazzara R, Monteagudo J, et al (1995) Thrombotic thrombocytopenic purpura/ hemolytic uremic syndrome: a multivariate analysis of factors predicting the response to plasma exchange. Ann Hematol 70:319-323

35. Rose M, Eldor A (1987) High incidence of relapses in thrombotic thrombocytopenic purpura. Clinical study of 38 patients. Am J Med 83:437-444

36. Rock GA, Shumak KH, Buskard NA, et al (1991) Comparison of plasma exchange with plasma infusion in the treatment of thrombotic thrombocytopenic purpura. Canadian Apheresis Study Group. N Engl J Med 325:393-397

37. Wong CS, Jelacic S, Habeeb RL, et al (2000) The risk of the hemolytic-uremic syndrome after antibiotic treatment of Escherichia coli O157:H7 infections. N Engl J Med 342:19301936

38. Sarode R, Gottschall JL, Aster RH, et al (1997) Thrombotic thrombocytopenic purpura: early and late responders. Am J Hematol 54:102-107

39. Yomtovian R, Niklinski W, Silver B, Sarode R, Tsai HM (2004) Rituximab for chronic recurring thrombotic thrombocytopenic purpura: a case report and review of the literature. $\mathrm{Br} \mathrm{J}$ Haematol 124:787-795

40. Gaddis TG, Guthrie TH, Jr, Drew MJ, et al (1997) Treatment of plasma refractory thrombotic thrombocytopenic purpura with protein A immunoabsorption. Am J Hematol 55:55-58

41. Bennett CL, Connors JM, Carwile JM, et al (2000) Thrombotic thrombocytopenic purpura associated with clopidogrel. N Engl J Med 342:1773-1777

42. Bennett CL, Weinberg PD, Rozenberg-Ben-Dror K, et al (1998) Thrombotic thrombocytopenic purpura associated with ticlopidine. A review of 60 cases. Ann Intern Med 128:541-544

43. Nagaya S, Wada H, Oka K, et al (1995) Hemostatic abnormalities and increased vascular endothelial cell markers in patients with red cell fragmentation syndrome induced by mitomycin C. Am J Hematol 50:237-243

44. Maslo C, Peraldi MN, Desenclos JC, et al (1997) Thrombotic microangiopathy and cytomegalovirus disease in patients infected with human immunodeficiency virus. Clin Infect Dis 24:350-355 